\title{
PRODUCTS OF CLOSED IMAGES OF CW-COMPLEXES AND $k$-SPACES
}

\author{
YOSHIO TANAKA AND ZHOU HAO-XUAN
}

\begin{abstract}
We give some characterizations for the products of closed images of CW-complexes to be $k$-spaces.
\end{abstract}

1. Introduction. C. H. Dowker [2] showed that not every product of two $\mathrm{CW}$-complexes is a CW-complex. Liu Ying-ming [6], assuming the continuum hypothesis $(\mathrm{CH})$, gave a necessary and sufficient condition for the product of two CW-complexes to be a CW-complex. The authors (independently, [13 and 16]) showed that Liu's characterization is equivalent to a certain set-theoretic axiom weaker than $(\mathrm{CH})$; see Corollary $3.2(1)$ in $\S 3$.

On the other hand, assuming $(\mathrm{CH})$, the first author [12] gave a characterization for the product of two closed images of metric spaces to be a $k$-space. G. Gruenhage [4] showed that this characterization is equivalent to an axiom weaker than $(\mathrm{CH})$.

In this paper, we shall give some analogous characterizations for the products of closed images of CW-complexes (or, closed images of spaces dominated by covers of connected, compact metric spaces) to be $k$-spaces.

Recall that a space $X$ is $k$-space if it has the weak topology with respect to the cover of all compact subsets $X_{\alpha}$; that is, a subset $F$ of $X$ is closed in $X$ whenever $F \cap X_{\alpha}$ is closed in $X_{\alpha}$ for every $\alpha$. Every CW-complex is a $k$-space.

We assume that all spaces are regular and all maps are continuous and onto.

2. Lemmas. Let us begin with some basic properties of $k$-spaces; for example, see $[\mathbf{3}]$. We shall omit the proofs.

LEMMA 2.1. (1) Every closed or open subset of a $k$-space is a $k$-space.

(2) Every locally $k$-space is a $k$-space.

(3) Every quotient (especially perfect) image of a $k$-space is a $k$-space.

Not every product of two $k$-spaces is a $k$-space, but the following sufficient conditions for the products are known. The assertions (1), 2(a) and 2(b) are respectively due to $[\mathbf{1}, \mathbf{8}$ and $\mathbf{1 6}]$. (MA) means Martin's Axiom.

LEMMA 2.2. (1) Every product of a locally compact space and a $k$-space is a $k$-space.

(2) Let $X$ and $Y$ have the weak topology with respect to covers $A$ and $B$ of compact subsets respectively. Then $X \times Y$ is a $k$-space if (a) $|A \cup B| \leq \omega$, or (b) (MA). Each element of $\mathcal{A}$ is metric, $|\mathcal{A}| \leq \omega$ and $|B|<c=2^{\omega}$.

Received by the editors July 13, 1983 and, in revised form, December 6, 1983.

1980 Mathematics Subject Classification. Primary 54D50, 54E60; Secondary 54B10, 54B15.

Key words and phrases. Weak topology, $k$-space, CW-complex, $B F(\alpha)$.

(C) 1984 American Mathematical Society $0002-9939 / 84 \$ 1.00+\$ .25$ per page 
For a cardinal number $\alpha$, let $S_{\alpha}$ (resp. $I_{\alpha}$ ) be the quotient space obtained from the topological sum of $\alpha$ convergent sequences (resp. $\alpha$ closed unit intervals $[0,1]$ ) by identifying all the limit points (resp. all the zero points).

Let ${ }^{\omega} \omega$ be the set of all functions from $\omega$ to $\omega$. For $f, g \in{ }^{\omega} \omega$, we define $f \geq g$ if $\{n \in \omega ; f(n)<g(n)\}$ is finite. Let $\mathbf{b}=\min \{\gamma$; there is an unbounded family $A \subset{ }^{\omega} \omega$ with $\left.|A|=\gamma\right\}$, where "unbounded" means in the sense of $\geq$ (so $A$ is unbounded iff no $f \in{ }^{\omega} \omega$ is $\geq$ every $g \in A$ ). By $B F(\alpha)$, we mean "b $\geq \alpha$ ". It is well-known that (MA) implies " $\mathbf{b}=c$ ".

As for the $k$-ness of the products of the spaces $S_{\alpha}, \mathrm{G}$. Gruenhage [4] showed the following result $((\mathrm{a}) \Leftrightarrow(\mathrm{c})$ of $(2)$ is stated in $[\mathbf{1 3}$ or $\mathbf{1 6}]) . \alpha^{+}$denotes the least cardinal greater than $\alpha$.

LEMMA 2.3. (1) $S_{\omega_{1}} \times S_{\omega_{1}}$ is not a $k$-space.

(2) The following are equivalent: (a) $B F\left(\alpha^{+}\right)$holds, (b) $S_{\omega} \times S_{\alpha}$ is a k-space, (c) $I_{\omega} \times I_{\alpha}$ is a $C W$-complex.

For a cardinal number $\alpha$, a space $X$ is $k_{\alpha}$ if it has the weak topology with respect to a cover $C$ of compact subsets with $|C| \leq \alpha$. Let us call a space $X$ locally $<k_{\beta}$, if each point $x \in X$ has a neighborhood whose closure is $k_{\alpha_{x}}$, where $\alpha_{x}<\beta$. Clearly, every locally $<k_{\omega}$-space (resp. locally $<k_{\omega_{1}}$-space) is precisely locally compact (resp. locally $k_{\omega}$ ).

Let $X$ be a space and $\left\{X_{\alpha}\right\}$ be a closed cover of $X$. Recall that $X$ is dominated ${ }^{1}$ by $\left\{X_{\alpha}\right\}$ if the union of any subcollection $\left\{X_{\beta}\right\}$ of $\left\{X_{\alpha}\right\}$ is closed in $X$ and the union has the weak topology with respect to $\left\{X_{\beta}\right\}$. Every CW-complex is dominated by the closed cover of all finite subcomplexes.

Recall that a space is Fréchet if, whenever $x \in \bar{A}$ then there is a sequence in $A$ converging to the point $x$.

Let us call a space $X$ a pre-S $S_{\alpha}$-space, if $X$ admits a perfect map onto the quotient space $S_{\alpha}$.

LEMMA 2.4. Suppose that $X$ is dominated by a closed cover of connected, Fréchet and $k_{\omega}$-spaces $X_{\alpha}$. Let $\beta$ be a regular cardinal. If $X$ contains no closed pre-S $S_{\beta}$-subspace, then $X$ is locally $<k_{\beta}$.

Proof. Suppose that $\beta \geq \omega_{1}$ and $X$ is not locally $<k_{\beta}$ at $x \in X$. Let $\mathcal{F}=\left\{X_{\alpha} ; x \in X_{\alpha}\right\}$, and let $N=\bigcup \mathcal{F}$. Since the union of any subcollection of $\left\{X_{\alpha}\right\}$ is closed in $X$, int $N$ is a neighborhood of $x$. But, $N$ is dominated by $₹$ and each element of $\mathcal{F}$ is $k_{\omega}$. Since, for each $\gamma<\beta, N$ is not $k_{\gamma}$, the set $N$ is not the union of $\gamma$ many elements of $\mathcal{F}$. Hence, we can choose a subcollection $\left\{X_{\gamma} ; \gamma<\beta\right\}$ of $\mathcal{F}$ such that $X_{\gamma}-X(\gamma) \neq \emptyset$, where $X(\gamma)=\bigcup_{\delta<\gamma} X_{\delta}$. But, for each $\gamma<\beta, X_{\gamma} \cup X(\gamma)$ is connected and $X(\gamma)$ is closed in $X$. So, $\overline{X_{\gamma}-X(\gamma)} \cap X(\gamma) \neq \emptyset$. Since each $X_{\gamma}$ is Fréchet and closed, there exist subsets $\left\{x_{\gamma} ; 1 \leq \gamma<\beta\right\}$ and $\left\{x_{\gamma n} ; n \in \omega\right\}$ of $X$ such that $x_{\gamma} \in X(\gamma), x_{\gamma n} \in X_{\gamma}-X(\gamma)$ and $x_{\gamma n} \rightarrow x_{\gamma}$. Then there is $f: \beta \rightarrow \beta$ such that $f(0)=0, f(\gamma)<\gamma$ with $x_{\gamma} \in X_{f(\gamma)}$ for $\gamma>0$. Now, since $\beta \geq \omega_{1}$ and $\beta$ is regular, by the Pressing-Down Lemma (for example, see [5, p. 80]) there is a subset $S \subset \beta$ with cardinality $\beta$ such that $f(S)=\beta_{0}$ for some $\beta_{0}<\beta$. Note that $x_{\gamma} \in X_{\beta_{0}}$ for $\gamma \in S$. Since $X_{\beta_{0}}$ is $\sigma$-compact, some compact subset $K$ of $K_{\rho_{0}}$

\footnotetext{
${ }^{1}$ For a cover $C$ of a space $X$, sometimes " $X$ is dominated by $C$ " means the same as " $X$ has the weak topology with respect to $C$ ". But these notions are distinct in this paper.
} 
contains a subset $\left\{x_{\beta(\gamma)} ; \gamma<\beta\right\}$ with cardinality $\leq \beta$, where $\{\beta(\gamma) ; \gamma<\beta\} \subset S$ and $\beta\left(\gamma^{\prime}\right)<\beta(\gamma)$ if $\gamma^{\prime}<\gamma$. Let $C=\{K\}^{\cup}\left\{L_{\gamma} ; \gamma<\beta\right\}$ and $X^{*}=\bigcup C$, where $L_{\gamma}=\left\{x_{\beta(\gamma)}\right\}^{\cup}\left\{x_{\beta(\gamma) n} ; n \in \omega\right\}$. Then $X^{*}$ is a closed subset of $X$ having the weak topology with respect to the cover $C$. Indeed, for $F \subset X^{*}$, let $K^{\prime}=K \cap F$ and each $L_{\gamma}^{\prime}=L_{\gamma} \cap F$ be relatively closed (hence, $K^{\prime}$ and each $L_{\gamma}^{\prime}$ are compact in $X$ ). Assume that $K^{\prime} \cup \bigcup_{\gamma<\delta} L_{\gamma}^{\prime}$ is closed in $X$ for all $\delta<\alpha$. Let $F_{\alpha}=K^{\prime} \cup \bigcup_{\gamma<\alpha} L_{\gamma}^{\prime}$. If $\alpha$ is isolated, then $F_{\alpha}$ is closed in $X$. So, let $\alpha$ be limit. Let $\mathcal{F}_{\alpha}=\left\{X_{\beta_{0}}\right\} \cup\left\{X_{\beta(\gamma)} ; \gamma<\alpha\right\}$. Note that $F_{\alpha}^{*}=\bigcup \mathcal{F}_{\alpha}$ is a closed subset of $X$ having the weak topology with respect to the cover $F_{\alpha}$. But, by the assumption, $F_{\alpha} \cap X_{\beta_{0}}$ and each $F_{\alpha} \cap X_{\beta(\gamma)}$ are closed subsets of $F_{\alpha}^{*}$. Hence, $F_{\alpha}$ is closed in $F_{\alpha}^{*}$, so is in $X$. Thus, by induction, $F=F_{\beta}$ is closed in $X$ (hence, in $X^{*}$ ). This suggests that $X^{*}$ is a closed subset of $X$ having the weak topology with respect to the cover $C$.

Now, let $Y$ be the quotient space obtained from $X^{*}$ by identifying all the points of the compact set $K$. Then $Y$ is the space $S_{\beta}$ which is a perfect image of $X^{*}$. Hence, $X$ contains a closed pre- $S_{\beta}$-subspace $X^{*}$. This is a contradiction. Hence, in case $\beta \geq \omega_{1}, X$ is locally $<k_{\beta}$. In case $\beta=\omega, X$ contains no closed pre- $S_{\omega}$-subspace. So, $X$ contains no closed pre- $S_{\omega_{1}}$-subspace. Thus, by the above argument, each $x \in X$ has a neighborhood whose closure $N_{x}$ is dominated by a countable cover of Fréchet $k_{\omega}$-subspaces, hence is a $k_{\omega}$-space with Fréchet "pieces". We remark that the space $S_{2}$ of Arens (see [3, Example 1.6.19]) is a pre- $S_{\omega}$-space. Thus, $N_{x}$ contains no closed copy of $S_{\omega}$ or of $S_{2}$. Thus, by the proof of Theorem 2.1 in [14] and by Corollary 1.4 in [14], the $k_{\omega}$-space $N_{x}$ with Fréchet "pieces" is locally compact. Hence, $X$ is locally compact, so that $X$ is locally $<k_{\omega}$. That completes the proof.

Every closed map is quotient. Then the following is easily proved, so we shall omit the proof.

LEMMA 2.5. Let $f: X \rightarrow Y$ be a closed map. If $X$ is dominated by $\left\{X_{\alpha}\right\}$, then $Y$ is dominated by $\left\{f\left(X_{\alpha}\right)\right\}$.

3. Results. Let $K$ be a CW-complex with cells $\{e\}$, and let $C(e)$ be the smallest finite subcomplex containing $e$; that is, $C(e)$ is the intersection of all the subcomplexes of $K$ which contain $e$. Then the following fact $(*)$ is well known:

(*) Any $C(e)$ is connected (for example, see [7]) and compact metric, also $K$ is a paracompact space $[\mathbf{9}]$ dominated by $\{C(e)\}$.

Now we are ready for the main theorem.

THEOREM 3.1. Let $X, Y$ be closed images of $C W$-complexes. (1) The following are equivalent: (a) $B F\left(\omega_{2}\right)$ is false, (b) $X \times Y$ is a $k$-space if and only if $X$ or $Y$ is locally compact, otherwise $X$ and $Y$ are locally $k_{\omega}$ (equivalently, locally separable).

(2) (MA). $X \times Y$ is a $k$-space if and only if $X$ or $Y$ is locally compact, otherwise one of $X$ and $Y$ is locally $k_{\omega}$ and another is $<k_{c}$. When $X=Y$, we can omit (MA).

ProOF. (1) By Lemmas 2.1 and 2.2, the "if" part of (b) holds. (b) $\Rightarrow$ (a) follows from Lemma 2.3(2). So we will prove the "only if" part of (b) from (a). By the above $(*)$ and Lemma $2.5, X$ and $Y$ are dominated by covers of connected, compact metric subspaces. Now suppose that neither $X$ nor $Y$ is locally compact. Then, by Lemma $2.4, X$ and $Y$ contain closed pre- $S_{\omega}$-subspaces. But, since $X \times Y$ 
is a $k$-space, by Lemmas 2.1 and 2.3(2), neither $X$ nor $Y$ contains a closed pre$S_{\omega_{1}}$-subspace. Thus, by Lemma $2.4, X$ and $Y$ are locally $k_{\omega}$. Since $X$ and $Y$ are dominated by covers of compact metric subspaces, any closed separable (resp. compact) subset of $X$ and $Y$ is contained in a countable (resp. finite) union of these compact metric subspaces, hence is $k_{\omega}$ (resp. separable metric). Thus $X$ and $Y$ are locally $k_{\omega}$-spaces iff they are locally separable.

(2) By Lemma 2.3, neither $S_{\omega_{1}} \times S_{\omega_{1}}$ nor $S_{\omega} \times S_{c}$ is a $k$-space. Thus, similarly we have the "only if" part. The "if" part follows from Lemmas 2.1 and 2.2.

Let us call a CW-complex $K$ locally $<\beta, \beta$ is a cardinal, if each $x \in K$ has a neighborhood which meets only $\alpha_{x}$ many closed cells $\bar{e}$, where $\alpha_{x}<\beta$.

Now, as is well-known, any compact subset of a CW-complex meets only finitely many cells. Also, every product of two CW-complexes is a CW-complex if and only if it is a $k$-space [11, Lemma 4.4]. Thus, by Theorem 3.1, we have the following result in $[\mathbf{1 6}]$ (for (1), also see [13]).

Corollary 3.2. Let $K, L$ be $C W$-complexes.

(1) The following are equivalent: (a) $B F\left(\omega_{2}\right)$ is false, (b) $K \times L$ is a $C W$-complex (equivalently, a $k$-space) if and only if $K$ or $Y$ is locally finite, otherwise $K$ and $L$ are locally countable.

(2) (MA). $K \times L$ is a $C W$-complex if and only if $K$ or $L$ is locally finite, otherwise one of $K$ and $L$ is locally countable and another is locally $<c$. When $K=L$, we can omit (MA).

COROLLARY 3.3. Let $Y$ be the closed image of a $C W$-complex.

(1) $Y^{2}$ is a $k$-space if and only if $Y$ is a locally $k_{\omega}$-space (equivalently, a locally separable space).

(2) $Y^{\omega}$ is a $k$-space if and only if $Y$ is a locally compact metric space.

PROOF. Since (1) follows from the latter part of Theorem 3.1(2), we will prove (2). Let $Y^{\omega}$ be a $k$-space. Since $\left(S_{\omega}\right)^{\omega}$ is not a $k$-space by [10, Theorem 1.3], $Y$ contains no closed pre- $S_{\omega}$-subspace by Lemma 2.1 . Thus, by Lemma $2.4, Y$ is locally compact. Hence the paracompact space $Y$ is locally metric, so $Y$ is metric.

REMARK 3.4. Theorem 3.1 and Corollary 3.3 hold if we replace "CW-complex(es)" by "space(s) dominated by connected, locally compact and separable metric subspaces". Indeed, such subspaces are $k_{\omega}$. Thus, using Lemmas 2.4 and 2.5, this replacement is possible.

\section{REFERENCES}

1. C. H. Cohen, Spaces with weak topology, Quart. J. Math. Oxford Ser. (2) 5 (1954), 77-80.

2. C. H. Dowker, Topology of metric complexes, Amer. J. Math. 74 (1952), 555-577.

3. R. Engelking, General topology, PWN, Warsaw, 1977.

4. G. Gruenhage, $k$-spaces and products of closed images of metric spaces, Proc. Amer. Math. Soc. 80 (1980), 478-482.

5. I. Juhasz, Cardinal functions in topology, Math. Centrum, Amsterdam, 1971.

6. Liu Ying-ming, $A$ necessary and sufficient condition for the products of $C W$-complexes, Acta Math. Sinica 21 (1978), 171-175. (Chinese)

7. A. T. Lundell and S. Weingram, The topology of $C W$-complexes, Van Nostrand Reinhold, New York, 1969.

8. E. Michael, Bi-quotient maps and cartesian products of quotient maps, Ann. Inst. Fourier (Grenoble) 18 (1968), 287-302. 
9. K. Morita, On spaces having the weak topology with respect to closed coverings. II, Proc. Japan Acad. 30 (1954) 711-717.

10. Y. Tanaka, Products of sequential spaces, Proc. Amer. Math. Soc. 54 (1976), 371-375.

11. __ Some necessary conditions for products of $k$-spaces, Bull. Tokyo Gakugei Univ. (4) 30 (1978), $1-16$.

12. __ , A characterization for the product of closed images of metric spaces to be a $k$-space, Proc. Amer. Math. Soc. 74 (1979), 166-170.

13. _ _ Products of $C W$-complexes, Proc. Amer. Math. Soc. 86 (1982), 503-507.

14. __ Metrizability of certain quotient spaces, Fund. Math. 119 (1983), 157-168.

15. J. H. C. Whitehead, Combinatorial homotopy. I, Bull. Amer. Math. Soc. 55 (1949), 213-245.

16. Zhou Hao-xuan, Weak topology and J. Whitehead's problem, (preprint).

DEPARTMENT OF MATHEMATICS, TOKyo GAKUgei University, Koganei-Shi, TOKYO, JAPAN

Department of Mathematics, Sichuan University, Chengdu, People's RepubLIC OF CHINA 